\title{
El bien común y el gobierno en Jacques Maritain
}

\author{
Mg. Franco Caballero \\ franco.caballero@live.cl \\ Municipalidad de Talca (Preuniversitario Junto a ti) \\ Mg. Javier Fattah \\ jfattah@filosofia.ucsc.cl \\ Universidad Católica del Maule
}

\section{RESUMEN}

No cabe duda que Maritain ha tenido una influencia considerable en las ideas políticas contemporáneas, en especial en la denominada social democracia. Es por esto que conviene revisar algunas de sus principales teorías respecto a las formas de gobierno y el bien común. Tratar las formas de gobierno y el bien común en Maritain lleva inevitablemente a tener que considerar aspectos metafísicos y antropológicos, en tanto, en el autor son estos aspectos los que de forma posterior determinan o por lo menos influyen de forma considerable en su teoría política.

Como hemos mencionado Maritain concede una importancia significativa a el aspecto antropológico y metafísico, en los relativo al primero, este se hace manifiesto en la noción de persona concebida como un todo, como una realidad sustancial, mientras que el aspecto metafísico puede verse en la idea de Dios como el Bien Común Supremo.

Palabras claves: bien común; maritain; gobierno; estado. 


\title{
The common good and government in Jacques Maritain
}

\begin{abstract}
There is no doubt that Maritain has had a considerable influence on contemporary political ideas, especially on the so-called social democracy. This is why it is convenient to review some of his main theories regarding the forms of government and the common good. To deal with the forms of government and the common good in Maritain inevitably leads inevitably to consider metaphysical and anthropological aspects, while in the author it is these aspects that subsequently determine or at least considerably influence his political theory.

As we have mentioned Maritain grants a significant importance to the anthropological and metaphysical aspect, in those relative to the first, this is made manifest in the notion of person conceived as a whole, as a substantial reality, while the metaphysical aspect can be seen in the idea of God as the Supreme Common Good.
\end{abstract}

Keywords: common good; maritain; government; state.

Artículo recibido: 05 octubre. 2021 Aceptado para publicación: 02 noviembre 2021

Correspondencia: franco.caballero@live.cl Conflictos de Interés: Ninguna que declarar 


\section{INTRODUCCIÓN}

No cabe duda que el bien común no solo tiene una importancia teórica, sino que también tiene implicaciones prácticas. De ahí que uno de los objetivos principales de la organización política sea la búsqueda del bien común de sus ciudadanos, o por lo menos, servir de guía para que la ciudadanía pueda alcanzar el conjunto de condiciones, tanto materiales como espirituales que permitan al sujeto alcanzar el bien y la paz dentro de la sociedad política. Es la existencia del bien común lo que da sentido a la sociedad, los hombres requieren de bienes que solo pueden ser alcanzados mediante un esfuerzo común, mediante colaboración entre los sujetos.

Dentro de la noción de bien común que posee Maritain es posible ver un fuerte componente metafísico y antropológico, siendo un claro ejemplo de esto la distinción que realiza del bien común, donde alude a la existencia de un bien común supremo, al que todas las cosas se encuentran orientadas. Este componente se hace manifiesto también en la noción de persona, donde establece una diferenciación entre la personalidad y la individualidad, perteneciendo la primera a un ámbito metafísico, mientras que la segunda guarda relación con la materia y queda como principio de diferenciación entre los hombres.

Al exponer su teoría política el autor hace manifiesto algunos de sus postulados antropológicos y metafísicos, tal es el caso de la idea de nación, donde se hace manifiesta la idea de la persona humana como un ser autónomo y libre, en tanto, reconoce que la obligación de la nación no es trasformar al sujeto, sino que comprenderlo. Dentro de esta misma línea, distingue entre la comunidad y la sociedad, donde las relaciones netamente sociales pareciesen darse dentro de la comunidad y no en la sociedad como tal, la que más bien tiene un carácter orientado hacia los aspectos económicos, pero no necesariamente comunes como ocurre en el caso de la comunidad.

Por último y en línea con la importancia que atribuye a la persona humana, Maritain atribuye al Estado un rol de "guía" que debe generar valores morales e incentivar ciertas conductas que permitan alcanzar la vida buena dentro de la comunidad y la sociedad. Ahora esto no implica un poder absoluto del Estado, sino que pareciese que este debe estar "sometido" a la constante revisión por parte de la ciudadanía, en tanto este interviene en sus formas de vida. 


\section{La idea de bien común en Maritain}

Maritain concibe el bien común como el conjunto de condiciones que deben desarrollarse para alcanzar un nivel de vida que permita la posesión del bien y la paz dentro de la sociedad. Nuestro autor suele usar el término obra común, el cual alude a las actividades tanto materiales como inmateriales que permiten a la sociedad alcanzar un régimen de justicia. La sociedad se encuentra orientada hacia la búsqueda de esta, en tanto, permite el pleno desarrollo de la persona humana, es decir, un desarrollo intelectual y moral:

"Sin embargo, la sociedad política está destinada esencialmente, en razón del fin terrenal que la específica, a desarrollar condiciones de medio que lleven a la multitud a un grade de vida material, intelectual y moral conveniente para el bien y la paz del todo, de tal suerte que cada persona se encuentre ayudada positivamente en la conquista progresiva de su plena vida de persona y de su libertad espiritual." (Maritain, 1999, pág. 175)

Es precisamente la existencia del bien común, de la obra común lo que da sentido a la existencia de la sociedad, sin la búsqueda de este elemento la conformación de la sociedad se quedaría sin uno de sus elementos esenciales.

Dentro del bien común es posible distinguir diversas clases, siendo el principal el Bien Común Supremo, es decir, Dios, Él es el sumo bien por antonomasia, en tanto, todas las cosas creadas se encuentran orientadas hacia Él; todo bien depende de este Bien Supremo. En lo relativo a los bienes comunes que han sido creados parece posible establecer ciertas distinciones entre ellos, siendo la primera de estas distinciones entre el bien común trascendental y el bien común natural. En el primer caso, es decir, en el bien común trascendental, podemos afirmar que este es el más perfecto de los bienes creados, ahora dentro del bien común trascendental Maritain distingue entre aquellos individuos que son bienaventurados y que por tanto ya han conquistado la vida divina; mientras que en un grado inferior se encuentran aquellos que están en el camino de la redención, siendo tarea de la iglesia "guiar" a este grupo hacia la bienaventuranza:

"Here, it is more than ever true that in different respects the person is for the community and the community is for the person. For there is for the Church a common work, which is continued redemption, to which each is ordained as the part to the work of the whole. But this common 
work is itself ordained to the personal good of each, to the union of each with God Himself, and to the application of the redeeming blood to each as a single person." (Maritain, 1972, pág. 85)

En lo relativo al bien común natural, podemos distinguir tres tipos; el bien común del cosmos, el bien común de la sociedad civil y el bien común de las inteligencias. El bien del cosmos hace alusión al orden de todo lo creado; en segundo lugar se presenta en bien común de la sociedad civil que a grandes rasgos puede ser definido como el conjunto de condiciones que permiten a los individuos alcanzar tanto su desarrollo moral como intelectual: "It is the good human life of the multitude, of a multitude of persons; it is their communion in good living." (Maritain, 1972, pág. 50). Por último, se encuentra el bien común de las inteligencias que a juicio de Maritain es el tesoro de la cultura que permite que los espíritus se comuniquen y unan entre sí "In the second way, the common good of the intellects is the intelligible treasure of culture in which minds communicate with one another.” (Maritain, 1972, pág. 51)

\subsection{Elemento metafísico y antropológico.}

Al igual que en Santo Tomás, la noción del bien común que posee Maritain tiene un fuerte componente metafísico y antropológico. En este sentido se debe señalar que la persona humana, es la única criatura que Dios ama por sí misma, solo el hombre se encuentra orientado hacia la gracia divina con libertad de correspondencia. Al ser la persona humana una criatura dotada de intelectualidad no participa de la esencia divina del mismo modo que el resto de los seres creados, posee una diferencia esencial y no de grado con respecto a los demás. Esta diferencia no solo tiene una importancia teórica sino, que también práctica, en tanto, el hombre no se encuentra frente a una subordinación absoluta en lo relativo al orden, a la organización política. El hombre posee una relación de amor directa con Dios, una relación entre persona y Persona "But there is only the wholly personal commandment of love: Thou (and not you) shalt love thy God with thy whole heart, thy whole soul, thy whole mind" (Jacques Maritain Raissa Maritain, 1960, pág. 82). A pesar de la existencia de esta relación personal no se debe caer en la idea de que Dios desea salvar a los hombres de forma aislada, en tanto, no existe salvación fuera de la iglesia, de la comunidad.

Dentro de la concepción de la persona humana nuestro autor establece una distinción entre la individualidad y la personalidad, la primera se corresponde con el principio 
metafísico de la materia, en el sentido que la materia es el "principio" diferenciador, es por la materia que los entes se diferencian. Ahora en un sentido moral la individualidad se asocia al egoísmo, busca la diferenciación de los demás, en tanto, los otros se perciben como una amenaza para la propia autonomía, en este sentido no se debe otorgar a la ida metafísica de individualidad una noción negativa, sino que esta negatividad se encuentra en nuestra existencia.

En lo relativo a la personalidad, lo primero que se debe tener en cuenta, es que desde una visión metafísica esta tiene su origen en la forma, lo que en el sujeto viene a ser el alma, al respecto señala Maritain:

"Metaphysically considered, personality is, as the Thomistic School rightly asserts, "subsistence," the ultimate achievement by which the creative influx seals, within itself, a nature face to face with the whole order of existence so that the existence which it receives is its own existence and its own perfection.”. (Maritain, 1972, págs. 40-41)

Es precisamente en el nivel metafísico de la personalidad donde se encuentra la causa de su posible perfección moral, es decir, el hombre no puede llegar a ser considerado una persona como tal si sus acciones ético-morales no se encuentran en concordancia con la realidad metafísica y espiritual que existe en el sujeto.

Definidos algunos de los elementos de la persona humana podemos afirmar que su ámbito de desarrollo natural es la sociedad. El surgimiento de la sociedad puede darse en virtud de dos razones fundamentales, en razón de su perfección y en razón de las necesidades. En el primer caso, es decir en razón de su perfección podemos argumentar que la persona humana es un ser abierto que tiende al diálogo y al amor y por tanto a la sociedad, puesto que inevitablemente el diálogo y el amor deben ser realizados con sus semejantes, es en virtud de esto que el sujeto tiende por naturaleza a la comunión "In our treatment of the characteristic features of the person, we noted that personahty tends by nature to communion." (Maritain, 1972, pág. 47). En lo que concierne a la razón de sus necesidades podemos afirmar que esta surge en virtud de su individualidad material, y por tanto, al no ser solo espíritu, sino que también materia el hombre requiere de los demás para su educación, alimentación, etc.

Es claro que dentro de la sociedad se dan distintos "todos", entendiendo que la persona humana se presenta como una realidad, como un todo. Dicho esto se debe tener en 
consideración que la totalidad de la persona humana tiene una unidad de carácter sustancial, es por esto que podemos afirmar que la persona posee un carácter unitario frente a la sociedad. No puede ser considerada como una parte de la sociedad, en tanto, hablar de una parte de la persona resulta opuesto al concepto mismo, tal como lo señala Santo Tomás "Et quia ratio partis contrariatur rationi personffi, ut dictum est, ideo anima separata non potest dici persona; quia quamvis separata non sit pars actu, tamen habet naturam ut sit pars.” (Tomás, 1933, pág. 207).

A diferencia de la persona que posee una unidad de carácter sustancial, la sociedad solo tiene una unidad de carácter accidental, en el sentido que está conformada por distintas partes que no necesariamente deben permanecer unidas a ella, un individuo puede abandonar la sociedad sin producir mayor perjuicio en esta.

Ha quedado de manifiesto que el hombre requiere de la sociedad para su pleno desarrollo, es en virtud de esto que resulta conveniente destacar un punto esencial de la filosofía de Maritain respecto a la sociedad, esta debe ser una sociedad de hombres libres. Entre las características que debe tener esta sociedad, se encuentra en primer lugar, el hecho de que debe ser personalista, es decir, su centro es la persona humana en toda su dimensión, entendiendo que si bien esta por su naturaleza vive en sociedad, la sociedad no es su fin último, sino que su fin último es la transcendencia, el encuentro con Dios "Así, la persona reclama la sociedad, y tiende siempre a trascenderla, hasta que, al fin, entra en la sociedad de Dios” (Segarra, 1998, pág. 384). Una segunda cualidad de la sociedad de hombres libres es su carácter comunitario, en este punto encontramos la problemática de cómo conciliar las diversas partes que se desarrollan en ella, como lograr conseguir el bien común terrenal sin perder de vista el hecho de que los individuos también persiguen sus propios bienes particulares.

Un tercer elemento de esta clase de sociedad es su carácter pluralista, siendo esta una derivación del carácter comunitario. La pluralidad se hace manifiesta en los diversos ámbitos de desarrollo, ya sea en las comunidades políticas, asociaciones académicas etc. es precisamente en este tipo de organizaciones donde los hombres ejercen su libertad de reunión, lo que a su vez les permite fijar objetivos comunes que ayuden a su plena realización, tanto individual como social. Como una última característica de la sociedad de hombres libres, se nos presenta su carácter teísta, esto teniendo en consideración que 
el fin último del sujeto es Dios, a esto se debe sumar el hecho de que la organización política se debe basar en el derecho natural.

\subsection{Propiedades y valores del bien común.}

Con anterioridad hemos señalado que el bien común puede ser entendido como el conjunto de condiciones que permiten al cuerpo social alcanzar una vida buena. En este sentido Maritain atribuye tres propiedades fundamentales al bien común. En primer lugar se nos presenta la moralidad, entendiendo que la organización política debe buscar el bien común y siendo el bien común un bien humano es necesariamente moral, es en virtud de esto que el bien común, la política y la moralidad van unidos; una política sin moralidad difícilmente podrá apuntar al bien común, por mucho que pueda garantizar utilidades y ventajas a los individuos. Al respecto señala Maritain: "no es solamente un conjunto de ventajas y utilidades, sino esencialmente rectitud de vida, buena y recta vida humana de la multitud" (Segarra, 1998, pág. 386). Dicho esto es licito afirmar que la existencia y relación entre la moral y el bien común es necesaria para lograr un verdadero bien común y no un mero utilitarismo.

Como segunda propiedad del bien común encontramos la redistribución. Esta propiedad alude básicamente a la exigencia moral de que el bien común llegue a cada una de las partes de la sociedad. Esta exigencia de redistribución no es solo moral, sino que también se da en virtud de la dignidad humana, en tanto, es entendida como un todo moral. En tercer lugar se presenta la autoridad, en el sentido que debe existir un grupo de individuos que guíen a los demás "es necesario que algunas en particular se encarguen de esa conducción y que las directrices que impidan, las decisiones que tomen al respecto, sean seguidas u obedecidas por los miembros de la comunidad" (Segarra, 1998, pág. 389).

El bien común no solo está conformado por propiedades, sino que también por valores, estos aluden propiamente al contenido del bien común, entendiéndose por tanto que el bien común no es una mera abstracción. Dicho esto debemos mencionar como primer valor la libertad, este valor resulta esencial, en tanto, el sujeto por su propia naturaleza tiende a la autonomía; un segundo valor es la justicia, esta es condición para la libertad o por lo menos para su conquista, en el sentido que al dar a cada uno lo que le corresponde el sujeto puede realizarse de forma plena y en libertad; en tercer lugar se encuentra la paz, siendo esta fruto de la justicia, en el sentido que mientras exista injusticia no puede haber paz, puesto que existe una perturbación de las normas morales. Finalmente encontramos 
la amistad cívica, esta se relaciona directamente con la redistribución, en tanto, la redistribución alcanza un carácter pleno cuando se realiza por la amistad cívica y no por la obligación legal. Ejercer la amistad cívica no es más que aplicar la virtud cristiana de la caridad.

\subsection{Bien común y Estado.}

Establecidos algunos elementos relativos a la forma del bien común y al tipo de sociedad que el autor considera como legítima resultará conveniente comenzar a revisar su idea de Estado-Nación y como los elementos anteriormente mencionados se comienzan a manifestar en su teoría política y propuestas sociales.

Es importante establecer en Maritain la idea del objeto común que subyace dentro del sistema de vida social, en relación a lo material y lo espiritual. Esta idea común recae en el ejercicio de las relaciones sociales, y por tanto cobra una significancia dentro de la imagen que tenemos sobre el Estado.

Existe, dice el autor, dos formas que interactúan dentro de una nación; dos formas que se comprenden desde lo orgánico y casi instintivo que posee una región social como un país, producto de sus costumbres, aspectos culturales, e incluso sentimientos y estados psicológicos comunes, a los cuales Maritain les llama Comunidad.

Por otra parte, el autor francés, menciona la idea de Sociedad, en la que le otorga un acervo progresista, en cuanto a que busca realizar o alcanzar tareas, que dependen de las voluntades y capacidades intelectuales de las personas; se determina en la Sociedad la razón y la fuerza moral, a diferencia de la Comunidad, que se determina por los instintos y las creencias.

Dentro de la Comunidad se establecen los grupos regionales, étnicos y las clases sociales; dentro de la Sociedad se establecen empresas comerciales, sindicatos obreros, cuerpos políticos y agrupaciones afines.

En ambos cuerpos, tenemos el objeto común que es la vida social, en la cual interactuamos y nos relacionamos, permitiendo establecer acuerdos y diferencias dentro de nuestros pensamientos, si consideramos el ámbito de la vida espiritual, como también permitiendo acciones que tienen como límites las delimitaciones de la justicia, en cuanto al ámbito de la vida material.

De esta manera Maritain estipula que la nación es una Comunidad, y no una Sociedad. "La nación tiene derechos, que no son más que los de las personas a participar en los 
valores humanos peculiares de una herencia nacional" ( (Maritain, El hombre y el Estado, 1974)

Si en una nación consideramos la aglutinación de distintos aspectos históricos y sociales, podremos comprender que tras ellos se encuentran núcleos de sentimientos comunes, que las personas que en esta comunidad participan manifiestan según las representaciones de la naturaleza y los instintos humanos otorgados de sus experiencias. Por tanto la idea de nación en Maritain, está necesariamente relacionada primero que todo, a los aspectos culturales, antes que a los aspectos progresistas, permitiendo solidificar la idea de que una nación que se construye debe intentar atender los sistemas de gobernanza a razón de la observación nacional y propia de dicha comunidad, antes que buscar y atender referencias en el extranjero.

La Nación no correspondería a intentar la transformación de las personas, sino que a su comprensión, pues declara el filósofo francés que la nación "no apela a la libertad y responsabilidad de la conciencia personal, sino que instila en las personas una segunda naturaleza" (Maritain, El hombre y el Estado, 1974).

La Nación, por tanto, es prudente comprenderla perteneciente y relativa a los antecedentes orgánicos de un país, en cuanto a determinación de lo que se es como región, o mejor dicho, lo que se ha llegado a ser. A diferencia de la idea del Estado, que corresponde a lo que podría ser, atendiendo el aspecto progresista de un país.

El Estado se concatena con la idea de Sociedad, pues busca atender las necesidades e intereses de las personas, lo que incluso pudiese cultivar los virtuosismos, propio de los aspectos que se atañen a los intereses morales. El Estado como Sociedad, es una manifestación de la razón del conjunto de personas, en cuanto conforme por diversas instituciones puede establecerse en la cima para administrar y aplicar la justicia cuando esta sea requerida.

Así menciona Maritain al Estado: “... es un organismo facultado para utilizar el poder y la coerción, integrado por expertos o especialistas en ordenamiento y bienestar públicos, un instrumento al servicio del hombre” (Maritain, El hombre y el Estado, 1974).

Si pensamos en los valores que deben regir el comportamiento de las personas, estos radicarán en la consagración de la idea de Nación, la cual prosperará más allá de los funcionamientos de las instituciones que componen el Estado. 
Pero si pensamos en la protección y el cuidado del tan anhelado bien común, es decir, de la responsabilidad por el ejercicio de la vida social, es preciso establecer que son las sociedades políticas, el Estado, las que propenderán a su óptima consagración. Maritain establece ciertos criterios para el bien común: la organización de una vida comunal, condiciones fiscales saneadas, aparatos militares fuertes, cuerpos de leyes justas, aduanas adecuadas e instituciones prudentes. Todas ellas, incluidas las conciencias de lo cívico, las virtudes políticas, el sentido del derecho, las libertades de todas las actividades, las prosperidades materiales, los esplendores espirituales, las rectitudes morales, entre otras son comunicables y perfectibles en pos de la libertad de las personas (Maritain, El hombre y el Estado, 1974).

Es propio del desarrollo y el progreso de la moral de las personas, el desempeño de los deberes que se ejercen dentro de un organismo como el Estado.

"Desde el último periodo del siglo XIX en adelante, se hizo necesaria la intervención del Estado para compensar el general desdén hacia la justicia y la solidaridad humana, que prevaleció durante las primeras fases de la revolución industrial. La legislación estatal sobre el trabajo es de por sí una exigencia del bien común”. (Maritain, El hombre y el Estado, 1974)

Es importante destacar la idea de Estado que tiene este autor, puesto que le otorga un sentido activo y generativo respecto a valores que construyen el bien común y el progreso moral de las personas, al considerar que este organismo permite posicionarse a favor del desarrollo humano y formativo de ellas.

No solo la legislación por la justicia y la solidaridad humana hacen del Estado una entidad formativa, sino que la legislación también funciona como un valedor del derecho del pueblo a que se pueda gobernar a sí mismo, por tanto, podremos decir que ante estas legislaciones debe someterse al yugo sostenedor de todo cuanto interviene en las vidas y formas de vida de las personas, que es el bien común.

\section{Es prudente aclarar qué entendemos por pueblo según Maritain:}

“(...) Considerando únicamente la significación política de la palabra, baste decir que pueblo es la multitud de personas que, unidas bajo leyes 
justas, por la mutua amistad, y para el bien común de sus humanas existencias, constituyen una sociedad política o un cuerpo político. La noción de cuerpo político entraña los miembros orgánicamente unidos que componen el cuerpo político (...) El pueblo está por encima del estado, no es para el estado, sino que el estado es para el pueblo”. (Maritain, El hombre y el Estado, 1974)

El principio de bien común en Maritain, sostiene la concepción del funcionamiento orgánico del pueblo como cuerpo político. Podríamos pensar que el bien común es la sangre del cuerpo del pueblo, en aspectos políticos.

\subsection{Noción de Derechos Humanos.}

Es importante destacar además la concepción de Jacques Maritain en cuanto a los Derechos Humanos, en las cuales establece que debiesen ser el punto de encuentro entre las distintas percepciones, creencias, ideologías que sostengan las personas. Podrán estar en desacuerdo en muchas cosas, pero su punto de encuentro deben ser los Derechos Humanos. A ellos se sostiene en cuanto a derechos en general, ya sean sociales o personales, determinando desde su perspectiva, como definitorio de las conclusiones prácticas que debiesen ser inamovibles para el inmenso desencuentro filosófico, cultural, religioso o existencial que existen entre tantas personas que habiten el mundo.

El problema que observa Maritain es el escepticismo que ha provocado el considerar que cada quien pudiese establecer sus derechos y a poder conducirse por la vida según lo que entienda y comprenda por sus propias leyes de vida. Este aspecto podría aproximarse al conflicto de la libertad actual que se defiende bajo el argumento de que cada quien pudiese actuar y pensar libremente según le plazca. El problema pues se suscribe a la idea de que existe un derecho natural, por tanto objetivo en perspectiva de Maritain, que es inherente a la existencia humana y que por tanto, debiese establecerse como el campo común para el desarrollo y preparación del bien común.

“¿Debemos intentar el restablecimiento de nuestra fe en los derechos humanos sobre la base de una filosofía auténtica? Esta filosofía verdadera de los derechos de la persona se basa en una idea real del derecho natural, contemplando desde una perspectiva ontológica y participando de las estructuras y requerimientos esenciales de la 
naturaleza creada por el Autor de la Existencia”. (Maritain, El hombre y el Estado, 1974)

Esto nos hace pensar que para este filósofo es necesario y prudente, para poder establecer los derechos humanos como campos comunes, de encuentro e incluso de base para las diferencias de pensamientos entre las personas, que estas puedan regirse por una ley superior. Esta ley superior, o también llamada filosofía auténtica, podrá unificar los criterios de los escepticismos planteados respecto a las verdades universales que permiten el desarrollo del bien común en las comunidades del mundo.

\section{CONCLUSIÓN}

Es importante concluir que la obra de Maritain convoca a desarrollar una búsqueda por el bien común para crecer como sociedad, en el establecimiento de hombres y mujeres libres, que puedan desarrollarse a plenitud, desde los ámbitos intelectuales y morales. Esto permitirá que la condición humana establezca evoluciones de consciencia en la medida que vaya armonizando sus relaciones personales, y considerando los campos de diferencia y encuentros para el desarrollo prudente de las leyes que nos puedan regir.

Por ello invita a establecer un encuentro en el campo común de una ley universal, que el autor establece como una verdad superior, en la cual en ella podamos sentar las bases de igualdad entre las personas para poder construir y edificar la vocación de la condición humana.

De esta manera podemos manifestar que el bien común de Maritain, habita en las concepciones de lo político aristotélico, al buscar un bien colectivo, al mismo tiempo que un bien personal, permitiendo que exista una real evolución y progreso de la especie humana.

Es prudente declarar además, que el pensamiento del filósofo francés posibilita la reflexión y supervisión de nuestras acciones, en el desarrollo de la ética y la moral, como un grado participativo que al mismo tiempo, es formativo, estableciendo cualidades de crecimiento y mejoramiento. De esta forma, el ser humano logra posicionarse en igual medida que un bien común superior, que propende al desempeño del bien, y que logra guiar y orientar las acciones y pensamientos humanos.

Por tanto no podemos dejar de destacar el pensamiento del bien común de Maritain, en tiempos donde el hombre se ha colocado en el centro con raíz de su voluntad, la cual activa las acciones y los pensamientos a placer personal, desbalanceando el propósito y 
sentido de lo social, en favor y diferencia de los privilegios que cada uno posea. Por tanto, el autor convocado, rinde inspiración al sentido de igualdad, solidaridad y conciencia colectiva que tanto nos falta.

\section{REFERENCIAS BIBLIOGRAFICAS}

Bars, H. (1962). Jacques Maritain En Nuestros Días. Barcelona: Estela.

Burgos, J. M. (1995). Cinco claves para comprender a Jacques Maritain. Acta Philosohica, 5-25.

Casquete, M. (2017). La ley natural en el pensamiento de Jacques Maritain. Pensamiento, 405-423.

Charles de Koninck; José Artigas Ramírez; Leopoldo Palacios. (1952). De la primacía del bien comun contra los personalistas : El princípio del orden nuevo. Madrid: Cultura Hispánica.

Daniel Barcala Juan Ramón Calo. (1987). El pensamiento de Jacques Maritain. Madrid: Cincel.

Illanes, J. L. (1975). Características del filosofar. Análisis del testimonio de Jacques Maritain. Anuario Filosofico, 193-245.

Jacques Maritain Raissa Maritain. (1960). Liturgy and contemplation. New York: Archbishop of New York.

Lang, P. (1995). La inteligencia ética. La propuesta de Jacques Maritain,. Paris: Editions scientifiques europées.

Maritain, J. (1967). De Bergson A Santo Tomás De Aquino. Buenos Aires: El club del lectores.

Maritain, J. (1969). Principios de una política humanista. Buenos Aires: Difusión.

Maritain, J. (1972). The person and the common good. Wisconsin: University of Notre Dame.

Maritain, J. (1974). El hombre y el Estado. Santiago, Chile: Editorial del Pacífico.

Maritain, J. (1999). Humanismo integral. Madrid: Editions Aubier.

McCool, G. (1994). The Neo-Thomists . Milwaukee: Marquette University Press.

Moreno, F. (1987). Actualidad de Jacques Maritain. Santiago: Marracci.

Mounier, E. (1938). Manifiesto Personalista. Londres: Longmans, Green and Co.

Naudon, C. (1948). El pensamiento social de Jacques Maritain. Santiago : Club de lectores. 
Plaza, J. (2007). Reflexiones sobre la ley natural en Jacques Maritain. Cordoba: Instituto Internacional Jacques Maritain.

Segarra, A. (1998). El bien común en la filosofía social de Jacques Maritain. Navarra: Universidad de Navarra.

Sousa, N. d. (1946). Princípios de uma política humanista. Rio de Janeiro: Agir.

Tomás, S. (1933). Scriptum super Sententiis Petri Lombardi. Paris: Lethielleux Éditions. 